\title{
Hybrid
}

Revue des arts et médiations humaines

$7 \mid 2021$

Le réseau créatif des langu.ages

\section{Guillemets et crochets : de l'influence de la syntaxe et de la grammaire des langages de code sur une poétique de la page}

\section{J. R. Carpenter}

Traducteur : Armelle Chrétien

\section{(2) OpenEdition}

\section{Journals}

Édition électronique

URL : https://journals.openedition.org/hybrid/691

DOI : 10.4000/hybrid.691

ISSN : 2276-3538

Cet article est une traduction de :

Straight quotes, square brackets: Page-based poetics inflected with the syntax and grammar of code languages - URL : https://journals.openedition.org/hybrid/684 [en]

Éditeur

Presses universitaires de Vincennes

Référence électronique

J. R. Carpenter, « Guillemets et crochets : de l'influence de la syntaxe et de la grammaire des langages de code sur une poétique de la page ». Hybrid [En ligne], 7 | 2021, mis en ligne le 08 avril 2021, consulté le 08 décembre 2022. URL : http://journals.openedition.org/hybrid/691 ; DOI : https://doi.org/10.4000/ hybrid.691

Ce document a été généré automatiquement le 14 avril 2022.

Tous droits réservés 


\section{Guillemets et crochets : de l'influence de la syntaxe et de la grammaire des langages de code sur une poétique de la page}

\section{J. R. Carpenter}

Traduction : Armelle Chrétien

\section{Introduction}

1 En anglais, le mot «translation » est composé de deux parties. Le préfixe trans-, qui signifie « de part en part », « au-delà » ou «à travers », est appliqué au mot -lation, du latin latio, signifiant "porté ", au sens de "charrié » ou de "maintenu ». Je dois d'emblée préciser que je m'intéresse davantage au trans- qu'à la -lation. Trans-, tel qu'il est ici employé, désigne un processus, un mouvement, un événement; -lation, un engagement, un fardeau, un labeur.

2 Le préfixe trans- peut être employé pour couvrir de grandes distances («transcontinental», "transatlantique») ou désigner un changement d'état («transmettre », «transférer », "transporter »). Il peut aussi, de façon plus abstraite, déployer une poétique de l'allée et venue, comme dans "transversal», du latin versus : littéralement " un tournant ", «tourner ». Chaque vers à son re-vers. Dans la versification grecque, la strophe est chantée sur scène dans un mouvement d'est en ouest. L'antistrophe lui répond d'ouest en est. Aucune des deux voix ne se tient en l'un ou l'autre de ces lieux. Elles appellent : de part en part, au-delà, à travers.

3 En tant qu'écrivaine, et en tant que migrante, je vis et je travaille dans des entre-deux : entre la langue anglaise et la langue française, entre les supports papier et numérique, entre la poésie et le monde universitaire. En anglais, on est souvent pris entre deux extrêmes : between a rock and a hard place, between the frying pan and the fire ${ }^{1}$. De même en traduction, une impression de dualisme persiste : entre le pré-texte et le post-texte, 
l'original et la copie. En français, « entre » est un espace tiers : l'entrespace. Cet article se propose d'habiter ce tiers espace comme lieu d'intersection dynamique. Il sera écrit en anglais mais pensera en partie en français.

4 Cet article est le prolongement d'une communication présentée lors de la journée d'étude Multilingual Digital Authorship, animée par Erika Fülöp à l'université de Lancaster le 8 mars 2018. Cette communication fut plus tard transformée en conférence présentée à la Heine Hause Literaturhaus de Düsseldorf à l'invitation de l'université d'été Literaturübersetzen: Translation and the Digital World, le 28 juin 2019. Je fais remonter dans le corps du texte la mention de ces contextes de présentation, autrement reléguée en note de bas de page, afin de souligner à la fois les cadres disciplinaires qui ont nourri ma pensée et les processus de traduction déjà à l'œuvre dans ce texte. Le présent article suit la traduction de textes entre contextes culturels et linguistiques, entre publics universitaires et non universitaires, entre formes papier, numériques et performatives.

5 Sous sa présente forme de publication numérique, cet article poursuit une exploration du mouvement des langues de part en part, au-delà et au travers des entrespaces. L'idée de l'« entre » renvoie ici à un troisième temps/espace/état, une texture, un événement, un déploiement palpable chargé de cacophonie, de liminalité, d'atemporalité et d'échanges asynchrones. Conformément à son sujet transitoire, ce texte alternera entre modes lyrique et analytique.

\section{Médiation}

6 Je me sers d'Internet comme d'un espace de création et de diffusion de textes expérimentaux depuis 1993. Ma première œuvre numérique date de 1995. J'ai depuis continué à explorer le potentiel sans cesse grandissant du Web en matière de multimodalité, d'intertextualité et d'articulation image-texte. J'ai également publié cinq livres. Une grande partie de ce que j'écris pour la page est le fruit d'opérations numériques. Une grande partie de mes écrits numériques n'atteignent jamais la page. Où réside alors cette écriture? Toute écriture numérique opère dans les entrespaces entre le côté serveur et le côté client, entre le code source et le résultat, entre le langage humain et informatique. Dès lors, toute écriture numérique est radicalement intemporelle - opérant simultanément dans le « là » du serveur et le « ici » de l'appareil local, entre le «si » du code source et le « alors » de l'écran.

7 Un texte qui s'affiche sur un écran d'ordinateur est toujours déjà un texte en traduction. Le poète anglais John Hall note ainsi qu'« un écran est un dispositif destiné à substituer une apparence à une autre... Un écran est une monstration et une dissimulation. Montre-t-il pour dissimuler ou dissimule-t-il pour montrer ${ }^{2}$ ». Avant que nous ayons eu le temps de méditer la question, le texte numérique crève l'écran. Il traverse sous forme de lumière le milieu élémentaire de l'air pour atterrir sur la surface humide de l'œil du lecteur. Il est aussitôt absorbé dans l'obscure intériorité du crâne.

Lorsqu'un texte numérique se déplace de part en part de réseaux mondialisés, à travers des câbles sous-marins, à travers les airs sous forme de signaux, à travers les airs sous forme de lumière, à travers l'œil jusqu'au cerveau pour en ressortir de nouveau, au-delà du corps sous forme de voix, repénétrant le corps sous forme de son, qu'est-ce qui est en même temps transporté ? Quelle trace du numérique persiste sur la page? 


\section{Ponctuation}

Comme son titre le suggère, cet article traite avant tout de questions de ponctuation soulevées par la traduction de textes poétiques entre contextes numérique et papier, avec une attention particulière portée à l'emploi des crochets et des guillemets. Dans Why do We Quote: The Culture and History of Quotation, Ruth Finnegan remarque ainsi : « Il est facile de penser que les guillemets vont de soi, sans être particulièrement attentif à leur présence ${ }^{3}$.» Les conventions employées de nos jours « ont connu de nombreuses évolutions au fil des siècles ${ }^{4}$ ». Les conventions de ponctuation varient grandement à l'intérieur de l'anglais et du français, mais aussi entre les deux langues. Elles sont plus inflexibles dans les langages de programmation. L'absence ou le placement fautif de la moindre virgule, du moindre crochet ou guillemet empêchera le programme de fonctionner. Aucune des marques de ponctuation nécessaires au fonctionnement fluide d'une page Web ne doit être visible à l'écran. Mais il peut y avoir des erreurs>.

L'usage non conventionnel des signes de ponctuation peut servir à des fins poétiques. Adam O'Fallon Price affirme ainsi que

la ponctuation, qui est essentiellement invisible et insignifiante pour les gens normaux, comme il se doit, est un sujet éminemment personnel pour les écrivains. Les points, les virgules, les deux points, les points-virgules, selon leur présence ou leur absence, leur ordre et leur emplacement, peuvent indiquer un développement, une conjecture, un doute, une finalité .

11 Que se passe-t-il lorsqu'une poète de la page papier est aussi une auteure de pages Web? Que se passe-t-il lorsque les conventions de ponctuation du code source apparaissent à l'écran? En quoi la syntaxe et la grammaire des langages de code peuvent-ils moduler les poétiques de la page et de l'écran?

\section{Entre ville}

En 2006, j'ai reçu une commande pour la création d'une nouvelle œuvre numérique à l'occasion du cinquantième anniversaire du Conseil des arts de Montréal. J'écris ici le titre de cette œuvre, Entre ville (2006), en italiques, car il s'agit bien du titre d'une œuvre indépendante, comme celui d'un livre. S'il avait été question d'un poème individuel inclus dans un recueil papier, en anglais le titre apparaîtrait entre guillemets virgules simples ou doubles, en fonction du guide stylistique de la publication : 'Entre Ville' ou "Entre Ville". Le titre est composé de deux mots français : « entre » et « ville », avec un jeu de mot sur le «centre-ville ». L'espace de l'œuvre n'est pas le centre de la ville mais plutôt les ruelles qui relient les rues du quartier de Mile End à Montréal. Entre ville est donc une ville entre: la ville entre nous.

Dans l'œuvre numérique, le titre apparaît entre guillemets français : « Entre Ville ». Du moins, c'est ce qu'on pourrait penser. À y regarder de plus près, les guillemets français ne sont pas représentés par leur caractère Unicode «». Ils sont en réalité composés des symboles « inférieur à » et "supérieur à », employés comme chevrons, « »>, laissant deviner la présence de la grammaire et de la syntaxe d'un autre langage derrière l'écran. Si l'on consulte le code source de la page, on s'aperçoit alors que le titre « Entre Ville $\gg>$ n'est pas du tout composé de caractères textuels. Il s'agit au contraire 
d'un fichier image entouré de guillemets anglais doubles à leur tour encadrés par des chevrons.

<img src="images/title_entreville_main.gif" width $=580 \quad$ height $=33 \quad$ border $=0$ alt="<<ENTRE VILLE >>">

Fig. 1

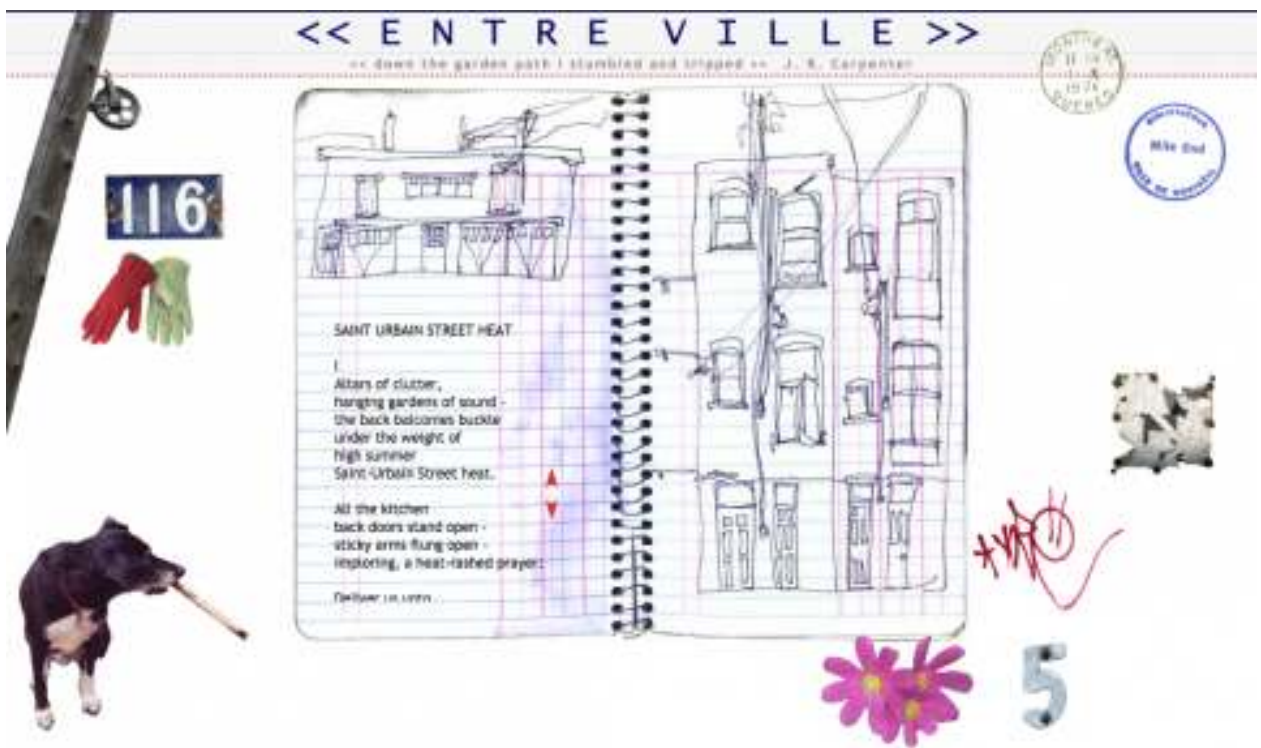

J. R. Carpenter, Entre Ville, 2006. [En ligne] http://luckysoap.com/entreville [consulté le 13 avril 2021]. une ville bilingue comme Montréal, mais cet exemple n'en soulève pas moins d'autres questions sur la ponctuation en traduction. Pourquoi les guillemets sont-ils représentés différemment en anglais, en français et en HTML ? Selon Finnegan :

La réponse semble tenir à un petit caractère graphique employé par les éditeurs de la Grèce antique pour attirer l'attention sur un élément notable au sein du texte.

En forme de chevron, on l'appelle le diplè (« double ») en raison des deux lignes qui dessinent son aspect cunéiforme. Sa principale fonction n'était pas, comme elle l'est aujourd'hui, d'isoler une citation, mais de servir de signe marginal pour attirer l'attention sur un aspect particulier du texte. Dans la tradition écrite occidentale, cette forme élémentaire semble avoir été le point de départ de la foule hétéroclite des formes modernes ${ }^{6}$.

L'emploi du double diplè dans le titre $<$ Entre Ville $>$ attire l'attention sur l'ambiguïté sémantique, l'imbrication de sens.

Outre ces éléments textuels, Entre Ville donne à voir un collage cacophonique d'images photographiques, de petites vidéos, de dessins, de graffitis, un timbre postal et des tampons de bibliothèque. Jay David Bolter et Richard Grusin qualifient la représentation d'un médium au sein d'un autre de « remédiation » et suggèrent que « la remédiation est un trait caractéristique du nouveau médium numérique ${ }^{7}$ ». Pourtant, le caractère directionnel du terme me paraît contestable, puisqu'il semble suggérer que nous évoluons inexorablement du papier vers le numérique. Dans le présent article, je souhaite démontrer que ces échanges sont de nature plus asynchrone. Ainsi mon premier roman, Words the Dog Knows (2008), remédiait-il le texte, le décor, les personnages et les dessins de mon poème numérique Entre Ville dans un roman papier. 
Je me suis enfin servie du roman papier comme support textuel pour une performance inspirée de l'œuvre numérique. Chacune des trois instances - papier, numérique, performance - remédient un poème initialement rédigé au stylo-plume sur du papier à lettres américain. Trans- apparaît ici comme un préfixe plus adéquat que re- en ce qu'il évoque les entrespaces du processus, celui d'inter- permettant à son tour d'embrasser l'écologie multimédia multilingue à laquelle renvoie ce corpus.

\section{GENERATION[S]}

Mon deuxième livre, GENERATION[S] (2010), est un recueil d'histoires très brèves générées par ordinateur et présentées en regard du code source du programme qui les a engendrées. Sur les pages qui contiennent les nouvelles, la ponctuation est représentée selon les conventions des publications papier. Sur les pages comprenant le code source, les conventions de ponctuation des langages de programmation ont été conservées. Dans l'espace numérique, ces textes sont variables: ils ne cessent de changer. Dans l'ouvrage imprimé comme à l'écran, chaque résultat n'est qu'une traduction parmi d'autres du code source, une transcription de l'exécution de JavaScript par un navigateur. L'œuvre papier expose ce processus numérique en l'interrompant, en le ralentissant.

GENERATION[S] propose aussi un méta-commentaire sur la composition de ces textes papier-numériques hybrides. Ce métacommentaire se présente notamment sous la forme de captures d'écran d'emails et de fils de commentaires Facebook, brouillant encore davantage les frontières entre papier et numérique. Contrairement à l'affirmation de Derrida selon laquelle « le livre, c'est à la fois le dispositif et le moment d'échéance qui nous obligent à interrompre le processus de l'ordinateur ${ }^{8}$ ", le livre papier apparait ici comme l'instantané d'un processus littéraire ouvert et continu, qui commencerait sinon à disparaître sitôt écrit. Dans This Little Art, Kate Briggs, traductrice des conférences posthumes de Roland Barthes, écrit : « Voilà ce qu'est l'acte d'écrire en tant que tel, par opposition au fantasme de l'écriture : une sorte d'arrêt, de halte ou d'immobilisation temporaire dans le mouvement de la culture'." Le livre papier GENERATION[S] sert aussi de machine à lire, de point d'entrée dans un processus compositionnel numérique, d'outil pour penser la traduction entre textes papier et numériques.

\section{The Gathering Cloud}

19 Mon troisième livre, The Gathering Cloud (2017), est la transmédiation d'une œuvre numérique antérieure du même nom (2016). Il existe aussi une petite publication papier du même texte en version zine. La chronologie ici importe peu. Ni le livre, ni le zine ni l'œuvre numérique ne sont l'original. Chacun est une -lation, une trace, transportée ou charriée d'un bord à l'autre à mesure que les idées derrière l'œuvre et les questions qu'elle pose évoluent à travers un continuum de formes. Peut-être convient-il dès lors de s'éloigner d'une réflexion sur une œuvre d'art individuelle pour penser à la place un corpus de textes. 
Fig. 2

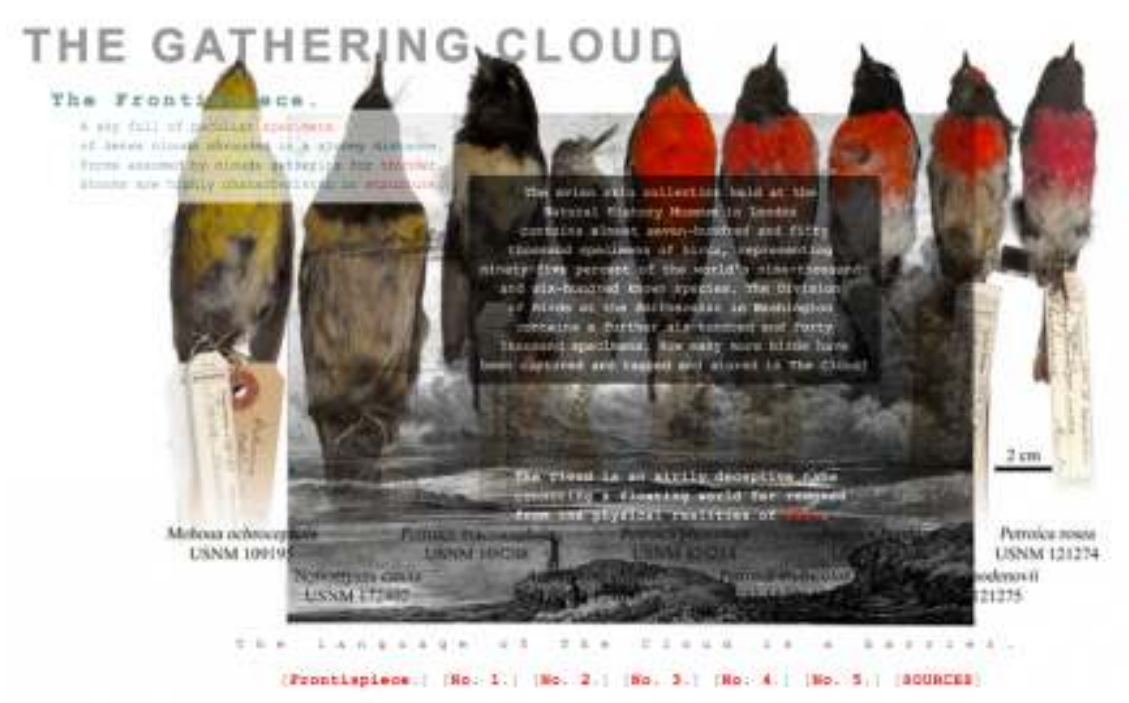

J. R. Carpenter, The Gathering Cloud, 2016. [En ligne] http://luckysoap.com/thegatheringcloud [consulté le 13 avril 2021].

En tant que corpus, The Gathering Cloud est aux prises avec la question de la transmutation. Là où la traduction d'une forme à l'autre suppose une équivalence entre les formes en jeu, la transmutation indique un changement soudain et/ou radical de forme. Ce corpus est né d'une question à la simplicité trompeuse : pourquoi parle-t-on de «nuage » pour désigner le cloud numérique ? La réponse à cette question exige la mobilisation de plusieurs médiums. Le livre papier retrace la transmutation du concept humain de nuage au fil de 2442 années de pensée, d'Aristophane et Aristote à Amazon et Google. Le poème numérique cherche à évoquer les conséquences environnementales du stockage sur le «cloud» en attirant indirectement l'attention sur la matérialité des nuages dans le ciel. Les deux sont habituellement perçus comme éphémères. Or, aucun ne l'est, bien au contraire.

21 Le livre papier et le poème numérique sont tous deux écrits en hendécasyllabes libres, ou en vers de onze syllabes. La plupart de ces vers sont des morceaux de textes préexistants, empruntées à des œuvres d'autres auteurs traitant des nuages ou du cloud. Aucun de ces «textes trouvés " n'est identifié comme tel par l'usage de guillemets. Aucun des auteurs n'est cité dans le corpus, même si la version papier et la version numérique contiennent chacune une liste de sources. Mckenzie Wark remarque à ce propos : «Le détournement est le contraire de la citation. Comme le détournement, la citation transporte le passé dans le présent, mais elle le fait entièrement dans un régime d'utilisation propre des noms propres $^{10}$. " Pour Gilles Deleuze et Félix Guattari, «le nom propre est l'appréhension instantanée d'une multiplicité11 ${ }^{1}$. C'est pourquoi « il n'y a pas d'énoncé individuel [...]. Tout énoncé est le produit d'un agencement machinique $^{12}$ ». Convoqués en tant qu'énoncés au sein d'un processus textuel numérique, ces fragments hendécasyllabiques sont reconstitués dans un présent qui marque aussi une rupture avec le présent, dans un nouveau régime d'énonciation, un régime dans lequel l'auctorialité est distribuée, où le texte n'est plus un objet mais un événement. Il en résulte une déterritorialisation, une politique incohérente, une politique qui selon Alexander Galloway «tend à dissoudre les liens institutionnels existants $^{13}$ ", y compris ceux de l'auctorialité. 


\section{An Ocean of Static}

La suite de cet article portera sur des exemples de poétiques de la page modulées par la syntaxe et la grammaire des langages de code au sein de mon quatrième livre, An Ocean of Static (2018). Davantage qu'un travail de traduction, je définis ce livre comme un objet transitionnel opérant entre langages, temps et espaces humains et informatiques, un instantané de processus ouverts et continus, une interruption, un lapsus, un glissement, un glitch.

Fig. 3

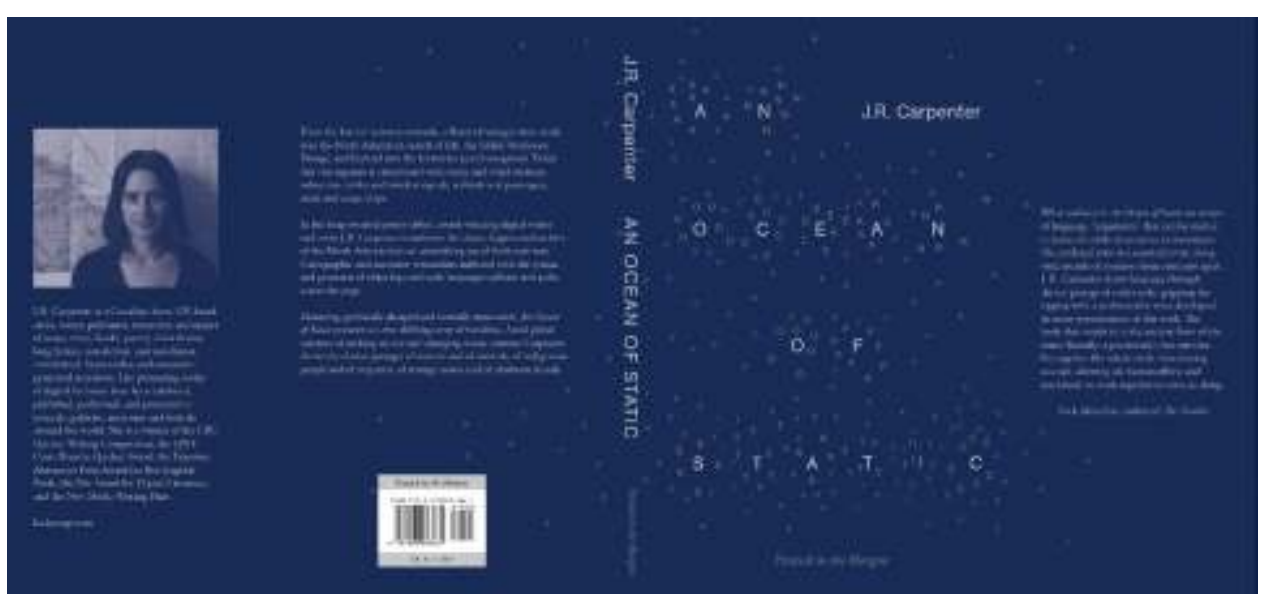

J. R. Carpenter, An Ocean of Static, Londres, Penned in the Margins, 2018.

23 La maison d'édition, Penned in the Margins, a présenté le livre comme mon premier recueil de poésie. Je ne sais pas vraiment ce que ça veut dire. Ce livre est le fruit d'un processus de composition itératif dans lequel l'acte d'écriture lui-même est toujours un événement, un déplacement dans le temps, et où ce qui est écrit est toujours en passe de devenir autre chose. Issus de processus numériques, variables, performatifs, incarnés et matériels, les textes de ce livre... je n'arrête pas de dire "textes ». Mon éditeur voudrait que je dise " poèmes ». Le résumé sur la quatrième de couverture dit :

Les jargons cartographiques et maritimes modulés par la syntaxe et la grammaire des livres de loch et des langages de programmation s'éparpillent et palpitent sur la page.

« S'éparpillent et palpitent », ça sonne bien. Mon éditeur est un vrai poète. J'allais dire « roulent et tanguent ».

Deleuze et Guattari ont eu cette célèbre formule : «Il n'y a pas de différence entre ce dont un livre parle et la manière dont il est fait ${ }^{14}$. » Ce livre évoque les générations de passages transatlantiques effectués par les courants, les navires, les paquets, les stylos et les passagers. Un lecteur attentif aura remarqué que j'ai tendance à enchaîner les phrases de la sorte: une affirmation suivie d'une longue liste de propositions. C'est parce que je parle d'un livre composé de variables, de chaînes de caractères, de tableaux, d'arguments :

Ce livre est fait de fragments - d'['autres livres', 'archives', 'livres de loch', 'codes source', 'JavaScripts', 'textes de performance']. 

certains aspects des règles de ponctuation qui régissent le code source. Lors de ma première réunion avec l'éditeur de Penned in the Margins, Tom Chivers, j'ai demandé à ce que tous les guillemets anglais simples qui figurent dans les passages comme ceux déjà cités demeurent des apostrophes droites " ' " dans le livre papier. La plupart des logiciels de traitement de texte et d'édition traduisent automatiquement les guillemets droits "' ' en guillemets courbes " '", conformément aux conventions typographiques héritées de l'imprimerie. Mais les arguments contenus dans An Ocean of Static relèvent d'un autre héritage textuel. Bien que la quasi-totalité du code source ait été perdue, le maintien des guillemets anglais droits et des crochets dans la version papier suggère au lecteur humain que ces arguments viennent d'ailleurs. Ils font en tant que tels appel à un autre mode de lecture.

31 En adaptant cette œuvre du temps informatique au temps humain, du support numérique au support papier, du JavaScript à un texte pour une performance, ce que j'ai produit n'est ni tout à fait une traduction, ni à proprement parler un résumé. Une acception plus large du concept de traduction - entre médiums, langues et contextes de présentation - est venu nourrir le processus compositionnel de cette œuvre.

\section{Etheric Ocean}

Le titre An Ocean of Static est une formule empruntée à un poème du recueil intitulé «Etheric Ocean » (p.119-134). Le poème papier est inspiré d'un poème numérique intitulé Etheric Ocean (2014) initialement commandé par Electronic Voice Phenomena, un projet de littérature expérimentale et nouveaux médias basé à Liverpool qui explore 
les approches contemporaines du son, de la voix, de la technologie et de l'écriture. La version numérique Etheric Ocean offre un relevé imprécis de sons, de signes et de distorsions nés de la difficulté de communiquer à travers le milieu dense, obscur et profond de l'océan. Pareils aux stations qui ponctuent le cadran d'une radio, diagrammes troubles, définitions changeantes, textes appropriés, associations nautiques et jeux de mots s'enchaînent au fil du long déroulement horizontal de la fenêtre du navigateur.

Sur la page d'accueil figure une première ligne de texte: «this sea is nothing in sight but isles ${ }^{19} »$. Une ligne de texte adjacente traduit le mot «isles » en un calembour visuel : «I'll(s)», comme plusieurs «I will ${ }^{20} »$. Une autre ligne encore demande : « [What will you do? $]^{21}$ ». La réponse est variable : la contraction « I'll » suivie d'un mot ou d'une expression sélectionnée de manière aléatoire à partir d'un tableau d'arguments piochés dans l'entrée « W » du Collins English Dictionary. Si cet océan n'est qu'îles à perte de vue, ces mots en « W» sont les vagues aqueuses - watery waves - qui viennent lécher inquiéter adorer - washing worrying worshiping - les rivages des îles. Bien qu'il ne s'agisse pas littéralement d'une traduction, le texte opère un processus similaire, proposant une débauche sans fin d'approximations, de possibilités, d'équivalences... aussi boiteuses ou absurdes qu'elles soient.

Fig. 4

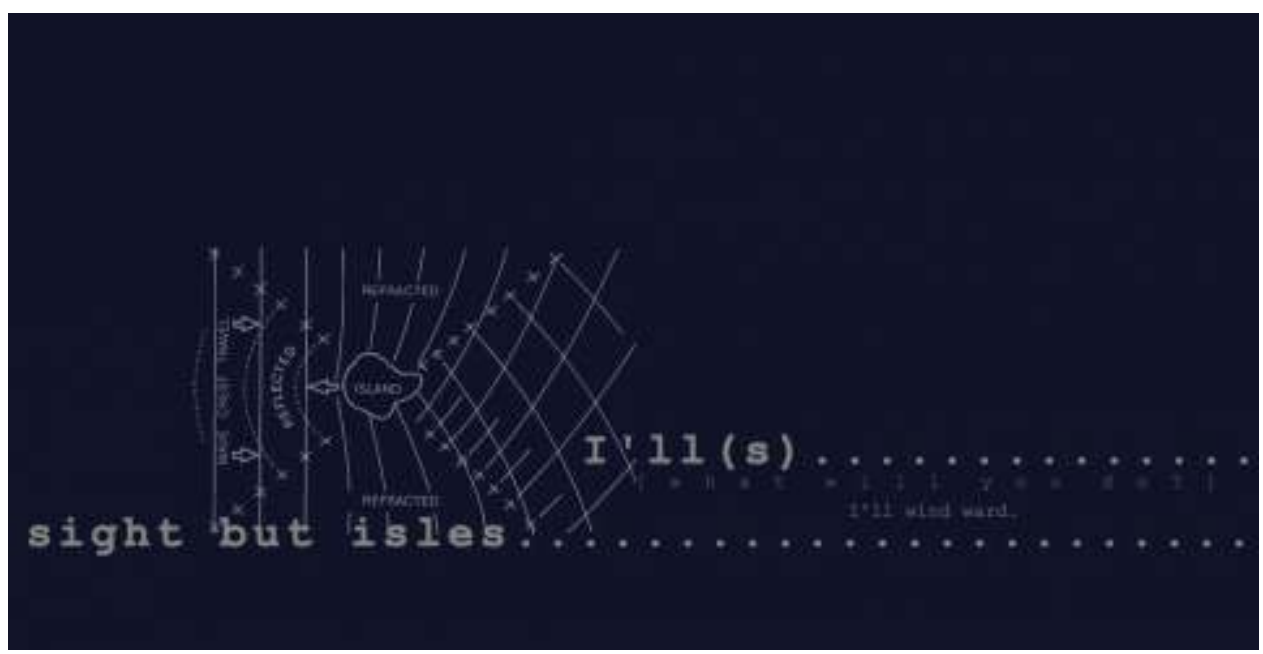

J. R. Carpenter, Etheric Ocean, 2014. [En ligne] http://luckysoap.com/ethericocean [consulté le 13 avril 2021].

En juin 2014, j'ai été invitée par Penned in the Margins à présenter une performance inspirée de la version numérique de Etheric Ocean à l'occasion d'une rencontre de poésie en partenariat avec le musée de l'eau Somerset House de Londres. Sur papier, j'ai imaginé un texte pour deux voix destiné à être lu pendant que le texte numérique serait projeté et déroulé de gauche à droite. Le texte imprimé se retrouva rapidement chargé de didascalies gribouillées en marge au crayon. Comme je devais lire de longues listes d'arguments copiées-collées à partir des tableaux JavaScript, je choisis de ['conserver la syntaxe JavaScript dans le texte imprimé', 'pour m'orienter à travers des phrases grammaticalement impossibles'].

Ce n'est pas un hasard si le mot « $\operatorname{script}^{22}$ » figure dans « JavaScript » : JavaScript est un langage procédural. Comme le texte d'une représentation, le JavaScript doit être écrit 
et lu dans un certain ordre pour être exécuté par le navigateur. Qu'est-ce qui est charrié, ou transporté, lorsqu'un texte conçu pour être exécuté par un navigateur est traduit en texte conçu pour être lu par un être humain ? À la différence de JavaScript, un lecteur humain pourra avoir besoin de coupures dans la phrase. Un retrait pourra venir indiquer une seconde voix plutôt qu'un élément de code imbriqué. Derrière l'écran, un crochet a un autre sens que sur la page ou sur scène. Les normes d'édition d'une pièce de théâtre varient d'un pays à l'autre: dans certains cas, les crochets pourront indiquer des didascalies. De même, JavaScript contient des instructions semblables à des didascalies, conçues non pas pour apparaître à l'écran mais pour déclencher une action. Dans d'autres cas, les crochets dans une pièce de théâtre peuvent indiquer une localisation: une indication qui invite l'acteur à remplacer un mot ou une expression du texte par quelque chose de signifiant pour le public. «Bonsoir [nom de lieu local] ! » devient : «Bonsoir, Paris!» Cet usage est semblable à l'utilisation de crochets dans JavaScript pour renfermer des tableaux.

Dans la version numérique de Etheric Ocean, la variable \{water\} peut uniquement convoquer un argument eau à la fois. La version papier utilise le JavaScript comme matériau brut à partir duquel créer un nouveau texte qui présente une partie seulement des arguments eau. En même temps, le texte imprimé traduit le processus de sélection aléatoire et continu exécuté par la machine dans un cadre temporel intelligible au lecteur humain :

I'll ['wade in', 'wait', 'wait a while'].

I'll ['walk in', 'walk away', 'walk on water'].

I'll ['water', 'down', 'fall', 'front', 'log', 'mark', 'meadow'].

I'll ['weaken', 'wean', 'wear', 'weather', 'warn', 'proof'].

I'll ['weather this storm'].

En 2014, j'ai adapté ce passage du texte de la performance pour le publier sous forme d'un poème indépendant, "this sea is nothing in sight but isles ", dans la revue en ligne 3:AM Magazine. La page Web présente le texte exactement comme le ferait une page papier, à une importante exception près. Un <iframe> intégré dans le code source affiche exactement le même fichier .js que la version numérique de Etheric Ocean présente sur mon serveur Web. Cette invitation programmatique d'une source extérieure interrompt l'espace-temps de la lecture humaine au moyen d'un processus de lecture/écriture qui opère dans un temps informatique.

En 2015, j'ai traduit la performance live de Etheric Ocean pour un contexte universitaire : le colloque Sound Studies: Art, Experience, Politics organisé pendant trois jours à l'université de Cambridge. De nombreux éléments du texte de la performance furent transposés dans le texte de la conférence, conçu pour être lu tout en faisant défiler l'œuvre de gauche à droite. Le texte de la conférence inscrit le texte numérique et celui de la performance dans un contexte historique et textuel plus vaste, notamment par le biais du dispositif de la citation. Le texte de la conférence nous apprend en effet qu' 'à l'occasion de son second voyage à la découverte du passage du Nord-Ouest, entrepris en 1586, le sieur John Davis de Dartmouth, dans le Devon, remarqua que “cette terre n'est qu'îles à perte de vue" ». Dans le texte numérique Etheric Ocean, publié 430 années plus tard, "cette terre » devient " cette mer ». Je définis ce changement lexical comme une traduction entre contextes, entre une performance poétique et une performance académique, entre l'océan arctique et un océan éthérique, entre un océan de glace et un océan de "statique », un océan de bruit. 
En 2016, j'ai été invitée à soumettre un texte pour Hybrid. A Journal of Arts and Human Mediations, revue en ligne de l'Université Paris 8 dans laquelle parait également le présent article. J'ai soumis à cette occasion une version du texte de la conférence «Etheric Ocean » adaptée pour être lue plutôt qu'écoutée. Ce texte fut ensuite traduit de l'anglais au français. Bien que Hybrid soit une revue en ligne, j'ai reçu par courrier un exemplaire papier de la traduction pour relecture. En plus de traduire les mots de l'anglais au français, la personne qui a traduit avait aussi traduit la ponctuation: les guillemets anglais droits requis par JavaScript pour décomposer le code source avaient été traduits en guillemets français :

J'[« interviens », « attends », « attends un moment »].

Je [« m'approche », « m'éloigne», « marche sur l'eau »].

Je [« eau », « en bas », « tombe », « devant », « journal », « marque », « prairie »].

Je [« faiblis », « sèvre », « use », « supporte », « préviens », « prouve »].

Je [« résiste à cette tempête »].

Les guillemets français sont inintelligibles pour JavaScript. De même la convention française consistant à laisser un espace entre la citation et sa marque de ponctuation empêchent JavaScript de fonctionner. Tout comme l'apostrophe courbe dans un syntagme comme "m'éloigne». Par-delà la syntaxe intraduisible de JavaScript, cet extrait pose d'autres problèmes de traduction. Le jeu de mot sur isle/I'll se perd, tout comme l'association avec l'eau à partir d'une longue liste de mots en «W ». N'ayant aucune idée de comment parvenir à une meilleure traduction du contenu littéraire du passage en question, ni même de sa faisabilité, je me suis contentée de barrer tous les guillemets français pour les remplacer par des guillemets anglais droits.

41 Poursuivons sur cet exemple. En 2015, j'ai présenté Etheric Ocean dans le cadre des rencontres de poésie The Other Room, à Manchester. À la suite de cette performance, j'ai été invitée à soumettre un extrait du texte pour l'anthologie papier publiée chaque année par The Other Room. Au cours d'un échange de mails avec l'un des éditeurs de l'anthologie, Tom Jenks, j'ai copié une ligne de texte à partir d'une maquette PDF qu'il m'avait envoyée et collée la phrase dans mon mail de réponse afin de lui faire voir une légère modification que je souhaitais apporter. Le morceau de texte en question était une phrase du texte imprimé, empruntée à l'œuvre numérique, indiquant au lecteur d'allumer les enceintes de son ordinateur.

If you can't hear sound here, it's possible that your computer or browser doesn't support the sound file format. Or, that you have your speakers turned off. Or, that you are a land mammal bending your ear to hear sounds deep under water ${ }^{23}$.

Le logiciel de courriel a débité une sorte de traduction du texte transformé en un long bloc de trois pages surchargé de texte superflu. Je cite ici un court extrait pour démontrer la nature de la répétition dans le texte traduit :

If you can't hear sound here, it's possible that your computer or browser doesn't support the file format. Or, If you can't hear sound here, it's possible that your computer or browser doesn't support the file format. Or, If you can't hear sound here, it's possible that your computer or browser doesn't support the file format. [...] Or, that you have your speakers turned off. that you have your speakers turned off.that you have your speakers turned off. that you have your speakers turned off.that you have your speakers turned off.that you have your speakers turned off. that you have your speakers turned off.that you have your speakers turned off.that you have your speakers turned off. that you have your speakers turned off.that you have your speakers turned off.that you have your speakers turned off. [...] Or, that you are a land mammal bending ear to hear sounds deep or, that you are a land mammal bending ear to hear sounds deep or, that you are a land mammal bending 
ear to hear sounds deep under water.under water. Under water.under water. under water. under water.under water. under

Contrairement à l'idée suggérée par l'expression " lost in translation », à savoir que le texte traduit est moindre, inférieur, qu'il manque de précision ou de clarté, cette traduction d'un logiciel à l'autre entraîne de nombreux gains. Ce nouveau texte, particulièrement volumineux, est né de ce que Nathan Jones appelle "une incision fortuite [glitch incision] [...] une faille dans un système qui révèle un autre système ${ }^{24} »$. Ici, le glitch révèle une profonde incompréhension entre le lecteur PDF Adobe Acrobat et le logiciel de courriel quant à ce qui constitue un espace entre mots, ce que signifie un retour à la ligne et comment combler au mieux ces failles dans la compréhension syntaxique. Jones ajoute ensuite: «La rencontre entre l'humain et le glitch est si essentielle à son pouvoir de nuisance que l'on pourrait en conclure que le glitch n'existe que sur le fond des attentes humaines ${ }^{25}$. » Je m'attendais à ce que la ligne que j'avais copiée soit la même que celle que j'allais coller. Je fus tellement ravie de ce nouveau texte débité par le logiciel de courriel que je le copiai aussitôt à partir de l'email, le collai dans un document Word, et commençai à l'envoyer comme fragment d'écriture conceptuelle à des revues littéraires.

Plutôt que de voir dans ce glitch une erreur insignifiante ou même un accident heureux, j'y vois l'aboutissement logique d'un processus d'écriture itératif et performatif. Le texte qui émerge de ce glitch est-il une traduction? Peut-être incarne-t-il davantage la transcription d'un échec de communication passager entre des médiums aussi différents que des mammifères terrestres, des ondes sonores, des ordinateurs et des océans.

En août 2016, le poète britannique S. J. Fowler et moi-même nous sommes servis de ce texte à glitch comme support d'une improvisation à deux voix présentée lors du South West Poetry Tour. Le texte parut la même année sous le titre « water.under » dans la revue canadienne The Goose ${ }^{26}$. En 2018, une version abrégée de "water.under » fut publiée, intégrée dans le corps de texte du poème «Etheric Ocean » compris dans An Ocean of Static.

Ce compte-rendu détaillé d'une traduction entre formes culturelles, linguistiques et médiatiques ne prend en compte que quelques lignes tirées d'un texte parmi d'autres du recueil. Il ne faut pas oublier que la plupart des autres textes - je veux dire " poèmes » - compris dans An Ocean of Static ont aussi connu des opérations similaires, se mouvant de part en part, au-delà et au travers d'archives papier, de réseaux numériques, d'océans, de l'air, d'yeux et d'oreilles. Qu'est-ce qui a été transporté ? Qu'est-ce qui a persisté ? Les questions sont ouvertes. Les réponses résident peut-être dans le livre lui-même. An Ocean of Static est un livre qui porte les marques de sa propre fabrication. 


\section{BIBLIOGRAPHIE}

Bolter, Jay David et Grusin, Richard, Remediation. Understanding New Media, Cambridge/Londres, MIT Press, 1999.

Briggs, Kate, This Little Art, Londres, Fitzcarraldo, 2017.

Carpenter, J. R., « Entre Ville », 2006. [En ligne] http://luckysoap.com/entreville/ [consulté le 5 avril 2021].

Carpenter, J. R., « Notes on the Voyage of Owl and Girl », 2013. [En ligne] http://luckysoap.com/ owlandgirl [consulté le 29 décembre 2019].

Carpenter, J. R., Etheric Ocean, 2014. [En ligne] http://luckysoap.com/ethericocean [consulté le 29 décembre 2019].

Carpenter, J. R., « this sea is nothing in sight but isles ", 3:AM MAGAZINE, 2014. [En ligne] https:// www.3ammagazine.com/3am/this-sea-is-nothing-in-sight-but-isles/ [consulté le 29 décembre 2019].

Carpenter, J. R., « water.under », The Goose, vol. 15, no. 1, 2016. [En ligne] https://scholars.wlu.ca/ thegoose/vol15/iss1/36/ [consulté le 29 décembre 2019].

Carpenter, J. R. et Fowler, S. J., « water.under », South West Poetry Tour, Schumacher College, Devon, UK, 2016. [En ligne] https://www.youtube.com/watch?v=YJdBccXYmEo [consulté le

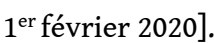

Carpenter, J. R., Ocean of Static, Londres, Penned in the Margins, 2018.

Deleuze, Gilles et Guattari, Félix, Mille Plateaux. Capitalisme et schizophrénie, Paris, Minuit, 1980.

Derrida, Jacques, Papier machine, Paris, Galilée, 2001.

Finnegan, Ruth, Why Do We Quote? The Culture and History of Quotation, Cambridge, Open Book Publishers, 2011.

Galloway, Alexander R., The Interface Effect, Cambridge, Polity, 2012.

Hall, John, Thirteen Ways of Talking about Performance Writing, Plymouth, Plymouth College of Art Press, 2007.

Jones, Nathan, « Glitch poetics : The posthumanities of error », in Joseph Tabbi (dir.), The Bloomsbury Handbook of Electronic Literature, Londres, Bloomsbury, 2017, p. 237-252.

O'Fallon Price, Adam, « Regarding the Em Dash », The Millions, 2018. [En ligne] https:// themillions.com/2018/01/regarding-the-em-dash.html [consulté le 27 juin 2020].

Wark, McKenzie, The Beach Beneath the Street. The Everyday Life and Glorious Times of The Situationist International, Londres/New York, Verso, 2011.

\section{NOTES}

1. Le français sur ce point n'est pas en reste, avec des expressions équivalentes telles que « entre l'enclume et le marteau », « entre deux feux », « entre Charybde et Scylla », etc. (N.d.T.).

2. John Hall, Thirteen Ways of Talking about Performance Writing, Plymouth, Plymouth College of Arts Press, 2007, p. 29. 
3. Ruth Finnegan, Why Do We Quote? The Culture and History of Quotation, Cambridge, Open Book Publishers, 2011, p. 79.

4. Ruth Finnegan, Why Do We Quote? The Culture and History of Quotation, Cambridge, Open Book Publishers, 2011, p. 80.

5. Adam O'Fallon Price, «Regarding the em dash», The Millions, 2018. [En ligne] https:// themillions.com/2018/01/regarding-the-em-dash.html [consulté le 27 juin 2020].

6. Ruth Finnegan, Why Do We Quote? The Culture and History of Quotation, Cambridge, Open Book Publishers, 2011, p. 86.

7. Jay David Bolter et Richard Grusin, Remediation. Understanding New Media, Cambridge/Londres, MIT Press, 1999, p. 45.

8. Jacques Derrida, Papier machine, Paris, Galilée, 2001, p. 161.

9. Kate Briggs, This Little Art, Londres, Fitzcarraldo, 2017, p. 191.

10. McKenzie Wark, The Beach Beneath the Street. The Everyday Life and Glorious Times of the Situationist International, Londres/New York, Verso, 2011, p. 40.

11. Gilles Deleuze et Félix Guattari, Mille Plateaux. Capitalisme et schizophrénie, Paris, Minuit, 1980, p. 51.

12. Gilles Deleuze et Félix Guattari, Mille Plateaux. Capitalisme et schizophrénie, Paris, Minuit, 1980, p. 51.

13. Alexander R. Galloway, The Interface Effect, Cambridge, Polity, 2012, p. 47.

14. Gilles Deleuze et Félix Guattari, Mille Plateaux. Capitalisme et schizophrénie, Paris, Minuit, 1980, p. 10.

15. J. R. Carpenter, An Ocean of Static, Londres, Penned in the Margins, 2018, p. 21 sq.

16. J. R. Carpenter, An Ocean of Static, Londres, Penned in the Margins, 2018, p. 23. Traduction : «Un hibou et une fille très ['aventureuse', 'curieuse', 'studieuse'] ['partirent', 'mirent voile', 'prirent la mer'] dans un ['bateau', 'esquif', 'navire', 'vaisseau'] ['vert bouteille', 'vert hanneton', 'vert pomme']; ».

17. J. R. Carpenter, «Notes on the Voyage of Owl and Girl», 2013. [En ligne] http:// luckysoap.com/owlandgirl [consulté le 29 décembre 2019].

18. Traduction : « ['aventureuse','ardente','courageuse','curieuse','déterminée', 'habile','ingénieuse','passionnée','rationnelle','rigoureuse','studieuse', 'sérieuse','travailleuse'] »

19. Traduction : « cette mer n'est qu'îles à perte de vue ».

20. Traduction : «Je vais".

21. Traduction : « [Qu'allez-vous faire?] »

22. On notera qu'en anglais, le terme « script » est employé pour désigner le texte d'une pièce de théâtre ou de toute autre forme de représentation, performance, etc. (N.d.T.).

23. Traduction : «Si vous n'entendez rien à ce stade, il est possible que votre ordinateur ou votre navigateur ne prenne pas en charge le fichier audio. Il se peut également que vos enceintes soient éteintes. Ou que vous soyez un mammifère terrestre qui tend l'oreille pour entendre des sons venus des profondeurs sous-marines."

24. Nathan Jones, "Glitch poetics: The posthumanities of error", in Joseph Tabbi (dir.), The Bloomsbury Handbook of Electronic Literature, Londres, Bloomsbury, 2017, p. 237.

25. Nathan Jones, "Glitch poetics: The posthumanities of error", in Joseph Tabbi (dir.), The Bloomsbury Handbook of Electronic Literature, Londres, Bloomsbury, 2017, p. 239.

26. J. R. Carpenter, "water.under», The Goose, vol. 15, n 1, 2016. [En ligne] https:// scholars.wlu.ca/thegoose/vol15/iss1/36/ [consulté le 29 décembre 2019]. 


\section{RÉSUMÉS}

Cet article étudie la traduction de textes à l'aune des contextes culturels et linguistiques, du lectorat, universitaire et grand public et des formes : imprimées, numériques et performées. Il s'intéresse en premier lieu aux questions de ponctuation qui surviennent lorsque l'on traduit des textes poétiques numériques en formats imprimés, et en particulier aux parenthèses et aux guillemets. S'appuyant sur une série de conférences, cet article, dans sa version actuelle de publication en ligne, mène une exploration continue du mouvement de la langue à travers, audelà et entre les espaces. L'idée d'« entre » est considérée ici comme un troisième temps/espace/ état, une texture, un événement, un déploiement palpable empreint de cacophonie, de liminalité, d'atemporalité et d'échanges asynchrones. Conformément à son sujet transitoire, cet article alterne entre les modes lyrique et analytique.

\section{INDEX}

Mots-clés : littérature digitale, poésie, performance, publication, ponctuation, traduction

\section{AUTEURS}

\section{J. R. CARPENTER}

J. R. Carpenter est une artiste, écrivaine et chercheuse qui travaille sur la performance et les médias print et numériques. Son travail a été présenté dans des musées, des galeries, des revues et des festivals dans le monde entier. Son œuvre en ligne The Gathering Cloud a remporté le New Media Writing Prize en 2016. Son recueil de poésie This is a Picture of Wind a été désigné par The Guardian comme l'un des meilleurs livres de poésie de 2020. En 2020-2021, elle est accueillie comme écrivaine en résidence à l'université d'Alberta. Site web : http://luckysoap.com. 